MS111.P01

\title{
Developing techniques for the structure determination of fatty esters
}

\author{
Prathapa Siriyara Jagannatha ${ }^{1}$, Cara Slabbert ${ }^{1}$, Manuel Fernandes ${ }^{1}$, Andreas Lemmerer $^{1}$ \\ ${ }^{1}$ Molecular Sciences Institute, School Of Chemistry, Univ. Of The Witwatersrand, Johannesburg, South Africa \\ E-mail: prathapa.siriyarajagannatha@wits.ac.za
}

Current interest in biodiesel sprouts, not only from environmental concerns, but in the recent past also from the global shortage of fossil fuels with concomitant upsurge of energy costs [1]. Biodiesel offers several advantages over diesel fuel, but its widespread use is hampered by some serious economical- and technical issues including physical properties. The main reaction in biodiesel production is transesterification with methanol or ethanol, in the presence of a catalyst, leading to a mixture of fatty acid esters (FAEs) (Fig. 1), the lengths of which (different values of $n$ ) are dependent on the source material. One of the main problems with biodiesels is linked to the relatively high melting points of the various FAE components and that the fuel solidifies at cold temperatures to form crystalline precipitates [2]. Little is known about the solid state structures and properties of these crystalline precipitates. Although various additives are already available to improve the cold-flow properties of biodiesels, and a partial solution can be obtained by mixing with mineral derived diesel, a systematic study of the crystallization of various pure components of biodiesel and their co-crystals may generate information related to melting point suppression of the various fuels by providing a solid-state chemistry model. Growing single crystals of the individual FAMEs, amenable for analysis by SCXRD, proved quite a challenge, since these compounds are either fluid liquid or solid waxes at room temperature (ca $25^{\circ} \mathrm{C}$ ). Single crystals of the liquid FAMEs (C4H9CO2CH3$\mathrm{C} 14 \mathrm{H} 29 \mathrm{CO} 2 \mathrm{CH} 3$ ) were grown with the aid of an Optical Heating and Crystallization Device (OHCD) via the zone melting method. In this technique, samples are loaded into a capillary, then cooled down to crystallization temperature using a cold stream of liquid nitrogen. Diffraction quality crystals are then created using a zone-melting technique where a small region of the capillary is heated with a CO2 IR laser to create a molten zone [3]. Here we present an overview of our structural analyses of these compounds

[1] Leung, D. Y. C. et al. (2010) Appl. Energy, 87, 1083.

[2] Ribeiro, N. M. et al. (2007), Energy \& Fuels, 21, 2433.

[3] Boese, R. et al. (1999), Angew. Chemie Int. Ed., 38, 988.

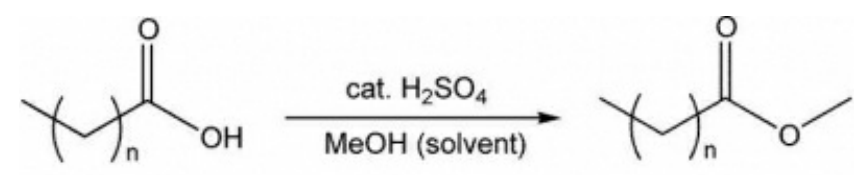

Fig. 1 General transesterification reaction scheme

Keywords: Biofuels, FAME, OHCD 